\title{
Phases of Polonium via Density Functional Theory
}

\author{
Matthieu J. Verstraete $e^{1,2, *}$ \\ ${ }^{1}$ Nano-Bio Group and ETSF, Departamento Física de Materiales, Universidad País Vasco UPV/EHU, \\ Centro Física de Materiales CSIC-UPV/EHU and DIPC, \\ E-20018 San Sebastián, Spain \\ ${ }^{2}$ Unité PCPM, Université Catholique de Louvain, Louvain-la-Neuve, Belgium
}

(Received 30 July 2009; published 20 January 2010)

\begin{abstract}
The thermodynamical properties of the main phases of metallic polonium are examined using density functional theory. The exceptional nature of the solid-solid phase transition of $\alpha$ to $\beta$ Po is underlined: it induces a lowering in symmetry, from cubic to rhombohedral, with increasing temperature. This is explained as the result of a delicate balance between bonding and entropic effects. Overall agreement with existing experimental data is good by state-of-the-art standards. The phonons of Po present Kohn anomalies, and it is shown that the effect of spin-orbit interactions is the inverse of that in normal metals: due to the nonspherical nature of the Fermi Surface, spin-orbit effects reduce nesting and harden most phonon frequencies.
\end{abstract}

DOI: 10.1103/PhysRevLett.104.035501

PACS numbers: 61.66.Bi, 61.50.Ah, 63.20.dk, 64.70.kd

A frantic race is underway to find exotic new phases of elemental solids under pressure [1-4]. With the advent of extreme diamond-anvil cell experiments with pressures beyond 1 MBar, a whole slew of new phases can be synthesized: with increasing pressure, the distance between atoms decreases, electron hybridization changes, and the nature of chemical bonds as well, changing coordination numbers and bonding directionality, orbital occupations, and so on. This is particularly dramatic in the recent demonstration that simple metals can become semiconducting if valence and core orbitals are forced to hybridize, through the application of pressure [2,3].

Comparatively much rarer are purely temperature induced solid to solid phase transitions. Although about half [5] of the elements present some form of solid-solid transition with $T$, most are in very limited pressure ranges, the critical pressure being more or less constant with $T$. Almost all of these transitions either increase or maintain the level of crystal symmetry (usually hexagonal to cubic, or cubic to cubic). This is intuitive, as temperature will increase interatomic distances, and tend to rotationally average orbital configurations, leading to high-symmetry (most often bcc) then liquid structures. The exceptions are the usual suspects: elements with complex bonding (boron, sulfur, phosphorous, and bismuth), and a few of those with delicate electronic shell effects, such as Ce or Dy. In these systems, an increase in temperature can give rise to a solid phase with lower symmetry.

Polonium also falls into this category. It is one of the strangest elements of the periodic table, and the only element which adopts the simple cubic (SC) [6] structure at ambient pressure ( $\alpha$-Po, joined by a few others such as Ca-III and As-II [7] at higher pressure). Upon increasing temperature, between 290 and $330 \mathrm{~K}$ [8], Po transforms to the $\beta$ phase, with a rhombohedral structure.
Despite its intriguing nature, experiments on Po are very difficult and rare, and little is known about its properties. Only a few laboratories in the world can synthesize Po in sufficient quantities to perform characterization. Because of their high radioactivity (with a half life of at most 100 years, depending on the isotope), samples heat and decay very quickly into $\mathrm{Pb}$ or $\mathrm{Bi}$.

By chemical analogy, Po should behave like Se and Te, its $4 p$ and $5 p$ cousins, and adopt a trigonal spiral structure at low T. However, it was shown recently $[9,10]$ that the SC structure is stabilized over the spiral structure by relativistic effects (though there is debate over whether the scalar relativistic (SR) or spin-orbit (SO) terms dominate-see comments to Ref. [9]). It was predicted that a simple rhombohedral structure would be stabilized under pressure. A qualitative explanation for the appearance of the SC structure is that the valence $p$ states do not hybridize with $s$, because of strong relativistic repulsion of the $s$ $[11,12]$. The $p$ states can therefore sustain purely rightangle bonding and an SC structure. Something similar probably happens in $\mathrm{Ca}$ and As, due to the relative (but not necessarily relativistic) changes in the $s$ and $p$ energy levels under pressure.

In this Letter, the vibrational and thermodynamical properties of crystalline Po are calculated from first principles. First, the effect of the spin-orbit interaction on the phonons of $\alpha$-Po is shown to be counterintuitive, hardening phonons and reducing the nesting of the Fermi surface (FS), the opposite behavior of simple metals. Thermodynamical quantities are calculated for the lowtemperature phase, and agreement with experiment confirms the precision of the first principles methods. Finally, the transition temperature of polonium is calculated at 0 pressure, comparing the Gibbs free energy of the different phases. Entropic and bonding effects are delicately bal- 


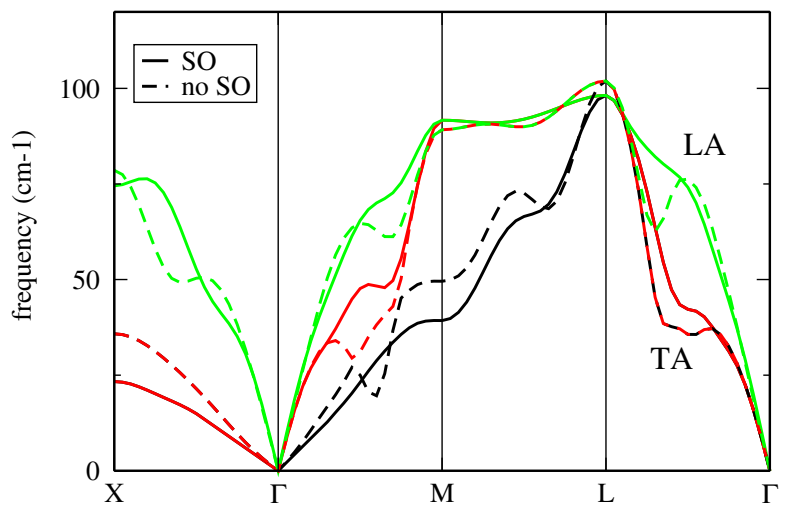

FIG. 1 (color online). The phonon band structure of SC polonium along high-symmetry directions of the Brillouin Zone, including the spin-orbit interaction (solid line), and without it (dashed line). Contrary to most normal metals, in polonium, spin-orbit decreases nesting, removes Kohn anomalies, and hardens most phonon frequencies.

anced and determine the stability of the $\alpha$ and $\beta$ phases as a function of temperature.

Density functional theory (DFT) [13] and the perturbation theory built on top of it (DFPT) [14] provide highly accurate calculations of the total energy and vibrational properties of materials from first principles. The implementation in the ABINIT package [15] is employed, with a plane wave basis (cut off at $435 \mathrm{eV}$ ) and pseudopotentials in the local density approximation [16]. Relativistic corrections are included through a pseudopotential of the HGH form [17]. The corrections are decomposed into SO and SR contributions. The calculations confirm the interpretation of Ref. [9] that the SR terms are sufficient to stabilize the simple cubic phase. Calculations without the SR relativistic terms will not be considered, because of the structure of the pseudopotentials used, and because for heavy elements this is quite unrealistic. It is the spin-orbit term which has the largest influence on the shape of the Fermi surface (which is the central quantity affecting phonon structure in metals). The DFPT formalism with SO is described in Refs. [18,19]. The thermodynamics of polonium are treated in the quasiharmonic approximation, fitting volume and temperature dependencies as described by Grabowski et al. [20] The Brillouin Zone is sampled with $16^{3} k$ points for electrons and $8^{3} q$ points for phonons.

The $\alpha$ phase of polonium is simple cubic, with a lattice constant of $3.345 \AA$. The theoretical equilibrium lattice constant is $3.335 \AA$, in good agreement, and will be used in the following. The phonon band structure of Po is shown in Fig. 1, with and without the effect of the SO interaction. In a simple metal, the Fermi surface departs only slightly from a sphere. Kohn anomalies (KA) [21] appear for phonons with reciprocal space vectors $\vec{q}$ which are nested in the surface, i.e., those $\vec{q}$ which connect many pairs of points on the surface. These phonons are screened particularly strongly by the electrons, which renormalizes their frequency. Distorting a spherical FS, in particular, because of the SO interaction, will always increase the nesting for certain $\vec{q}$ (sometimes dramatically as in $\mathrm{Pb}$ [19]). In polonium, the opposite happens: due to the orbital nature and SC crystal structure, the Fermi surface is more cubic than spherical (see Fig. 2). As a result, the deformations due to the SO interaction will decrease the nesting, instead of increasing it. The small spherical hole pocket around $\Gamma$ is also removed when the SO interaction is added. These nesting features, and their removal by the SO interaction, were first analyzed with dielectric response properties in Ref. [10]. The result is quite clear in the phonon bands of Fig. 1: when including the SO interaction (going from dashed to full lines), the dips due to Kohn anomalies are reduced, and the longitudinal (LA) frequency increases. The transverse (TA) frequencies increase or decrease in different regions of the Brillouin Zone, but also present KA which are reduced by the spin-orbit interaction. From the vibrational calculations for the $\alpha$ phase with and without $\mathrm{SO}$, it is clear that inclusion of full relativistic effects is crucial to the correct representation of the thermal properties of Po. On this basis, the thermodynamical behavior of $\alpha$ and $\beta$ Po (and their relative stability) will be examined in the following including both SR and SO terms.

Thermodynamical quantities are calculated from the phonon bands of the bulk. To capture thermal expansion, full phonon calculations are also performed for a volume $3 \%$ larger than equilibrium, and the mode-Grüneisen parameters are extracted (see, e.g., Ref. [20]). The volumetric thermal expansion of Po is shown in Fig. 3 (solid line); it is initially negative, which is found in many materials. For low temperatures, the mode behavior near the Brillouin Zone center is crucial. The contribution from the smallest $\vec{q}$ vectors (up to $1 / 8$ of the Brillouin Zone) is therefore fit to explicit calculations at small $\vec{q}$, and integrated numerically.

The principal thermodynamical quantities for which there is experimental data are the thermal expansion coefficient and the heat capacity. Brocklehurst et al. [22] found a thermal expansion coefficient of $23 \pm 1.5 \times 10^{-6} \mathrm{~K}^{-1}$ at $298 \mathrm{~K}$. Here, first principles calculations give $16 \times$ $10^{-6} \mathrm{~K}^{-1}$ which is in reasonable agreement. The tabulated heat capacity of polonium at $300 \mathrm{~K}$ is $C_{P}=$ $26.4 \mathrm{~J} \mathrm{~mol}^{-1} \mathrm{~K}^{-1}$ [23], quite close to the ab initio value of $24.9 \mathrm{~J} \mathrm{~mol}^{-1} \mathrm{~K}^{-1}$ found here. The correction due to SO
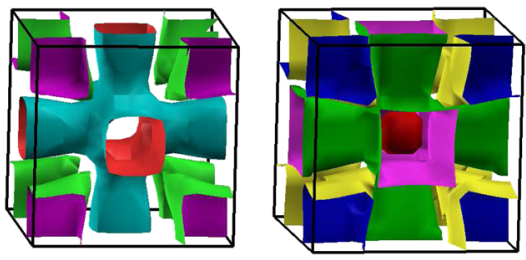

FIG. 2 (color online). Fermi surface of simple cubic Po, with (left) and without (right) the spin-orbit interaction. The SO interaction reduces the nesting of the Fermi surface, which in turn reduces the Kohn anomalies in Fig. 1. 


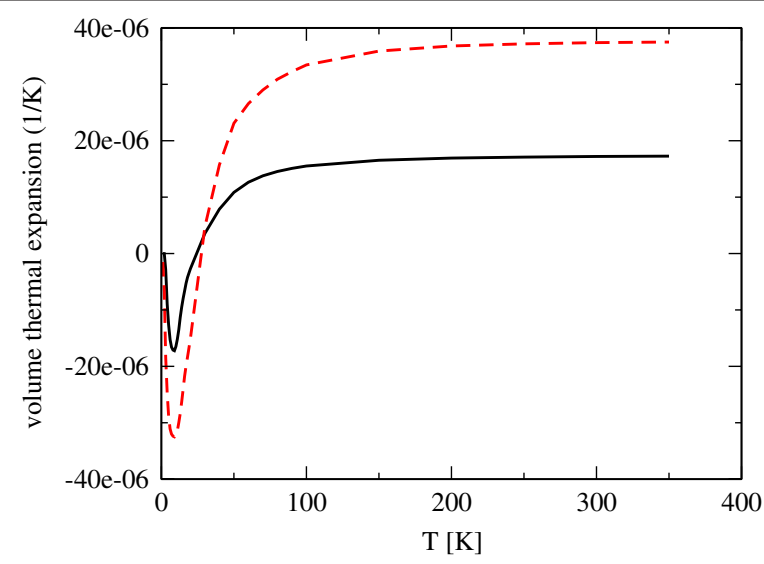

FIG. 3 (color online). Volumetric thermal expansion of Po as a function of temperature. Solid line: $\alpha$ phase. Dashed line: constant angle rhombohedral $\beta$ phase. Experiments [6,22] give $23 \pm 1.5 \times 10^{-6} \mathrm{~K}^{-1}$ at $300 \mathrm{~K}$ for $\alpha \mathrm{Po}$, and $50 \pm 25 \times$ $10^{-6} \mathrm{~K}^{-1}$ for $\beta$ Po.

coupling is slight $\left(+0.1 \mathrm{~J} \mathrm{~mol}^{-1} \mathrm{~K}^{-1}\right)$. A fit to Murnaghan's [24] equation of state for $\alpha$-Po gives a bulk modulus $B=39.45 \mathrm{GPa}$, volume $250 \mathrm{bohr}^{3}$, and $B^{\prime}=4.89$.

We now turn to the thermodynamics of $\beta$-Po, and the explanation of why this lower-symmetry phase is favored at higher temperatures, examining the contributions to the free energy which stabilize $\alpha$ or $\beta$ Po at different temperatures. The beta phase of polonium is rhombohedral, with a lattice constant of $3.359 \AA$ and an angle of $98^{\circ} 13^{\prime}$. It corresponds to a cube slightly compressed along the (111) diagonal, and possesses fairly low symmetry. As noted in previous studies, the structure is intermediate between the SC and a body-centered-cubic phase. For compressed volumes, first one rhombohedral phase becomes stable, for volumes smaller than about $0.93 V_{0}$ (calculated pressure of $1 \mathrm{GPa}$ ), with an angle larger than $90^{\circ}$. At compressions below $90 \%$ (1.5 to $2 \mathrm{GPa}$ ), a second phase is also stabilized, with an angle smaller than $90^{\circ}$ (as in Ref. [9]). Given the good performance of the DFT methods for the SC phase, the experimental unit cell is used to compute the phonon bands of $\beta$-Po, and the equation of state is fit for a constant rhombohedral angle (giving $B=40.15 \mathrm{GPa}$, volume 247 bohr $^{3}$, and $B^{\prime}=4.49$ ). Thermal expansion effects are then taken into account as for the $\alpha$ phase. This will be a very good approximation for volumes and temperatures close to our reference point, which is the experimental

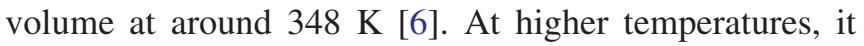
can be expected that the rhombohedral angle will change slightly. The thermal expansion for the fixed-angle beta phase is reported in Fig. 3 (dashed line), and is consistent with existing experimental data, being higher than (about twice) that of the $\alpha$ phase.

The phonon band structure of $\beta$-Po is shown in Fig. 4, along the same high-symmetry directions as in Fig. 1 (the $\mathrm{SC}$ nomenclature is used for simplicity). The phonons are

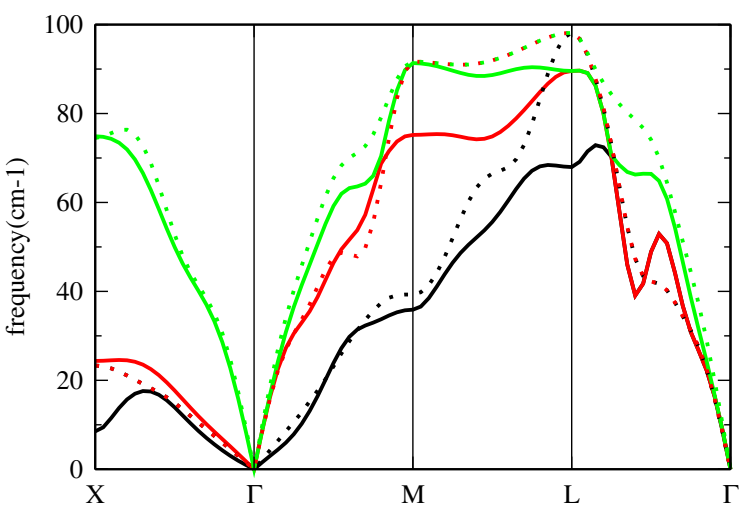

FIG. 4 (color online). The phonon band structure of $\beta$ polonium along high-symmetry directions of the Brillouin Zone. The $\mathrm{SC}$ phonons are included for comparison (dotted lines).

calculated at $0 \mathrm{~K}$ (with thermal expansion as for the $\alpha$ phase), and would change somewhat due to anharmonic effects (see, e.g., Ref. [25]). Work in this direction is ongoing, but the current agreement with experiment suggests that anharmonic effects are small. Frozen phonon calculations, carried out at zone boundary points, confirm this. The reduced anharmonic force constants are calculated, as defined in Ref. [26]. For both $\alpha$ and $\beta$ phases, the reduced third order constants are negligible $\left(k_{3} \leq 10^{-8}\right)$ and the fourth order constants $\left(k_{4} \leq 0.005\right)$ are 1 to 2 orders of magnitude smaller than in the case of doped diamond. It has been checked that lowering the electronic temperature does not lead to unstable phonons in the dip between $L$ and $\Gamma$, such that the thermodynamics described by the $0 \mathrm{~K}$ phonons is physical.

The free energy is calculated for $\alpha$ and $\beta$-Po, adding the ground state energy to the vibrational and electronic free energies. The free energies are calculated at a second volume $V^{\prime}$, and an additional correction is added, taking the thermal expansion into account (bottom panel of Fig. 5): $\Delta F_{x}(T)=\left[F_{x}\left(V^{\prime}, T\right)-F_{x}\left(V_{0}, T\right)\right]\left[V(T)-V_{0}\right] /$ $\left(V^{\prime}-V_{0}\right)$ where $x$ is vibrational or electronic. The volume $V(T)$ is obtained by inverting the volume dependency in the Murnaghan terms of $P=-\partial F_{\text {tot }} / \partial V=0$. Inserting $V(T)$ in the equation of state gives the "internal energy." The differences in the total free energy and its components, between the $\alpha$ and the $\beta$ phases, are shown in Fig. 5. In Ref. [9], the relativistic stabilization of $\alpha$-Po is calculated to be $0.15 \mathrm{mHa}$. Up to $\sim 600 \mathrm{~K}$, the internal energy difference between the phases (including the relativistic stabilization and the volume expansion for both) is ca. $0.4 \mathrm{mHa}$. At $350 \mathrm{~K}$, the vibrational entropy difference is also of the order of $0.4 \mathrm{mHa}$, but favors the $\beta$ phase. The other terms are small. The transition can be explained by the different vibrational entropy terms, favorable to $\beta$-Po, overcoming the internal energy difference. The basic phase diagram for heavy elements of the Se-Te column is thus restored at higher temperatures, once the relativistic stabilization of the $\alpha$ phase is overcome. The symmetry lowering can 


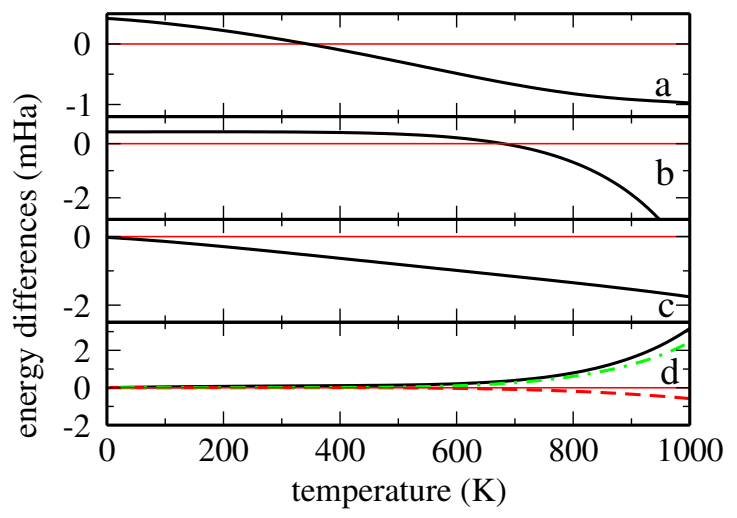

FIG. 5 (color online). Free energy differences (mHa) between $\beta$ and $\alpha$ polonium, as a function of temperature (Kelvin). Negative values favor $\beta$ polonium. (a) total free energies. (b) internal energy. (c) vibrational free energy at constant volume $F_{\text {vib }}$. (d) volume-correction $\Delta F_{\text {vib }}$ (solid line), electronic free energy $F_{\text {el }}$ (dashed line), and volume-correction $\Delta F_{\text {el }}$ (dot-dashed line). It is the vibrational free energy terms and the volume corrections that determine the transition $T_{c}$ from $\alpha$ to $\beta$ Po.

therefore be explained as the "normal" series of trigonal phases (trigonal spiral then $\beta$-Po then $\mathrm{BCC}$ ) being disrupted by relativistic bonding effects at temperatures low enough for the bonding and entropic terms to be comparable.

Comparing the full Gibbs free energy $(P=0)$ of $\alpha$-Po and $\beta$-Po determines the transition temperature $T_{c}$. The theoretical $T_{c}$ (at 0 pressure) from the $\alpha$ to the $\beta$ phase is $350 \pm 50 \mathrm{~K}$. The error is estimated from the convergence of the free energy fit parameters, and does not take into account anharmonic corrections. Experimental data give a range of $T_{c}$ from 290 to $330 \mathrm{~K}$. Omitting the thermal expansion or the free energy terms stabilizes $\alpha$-Po for all $T$.

In summary, this Letter presents an ab initio study of the different thermodynamical phases of crystalline Po. The effect of spin-orbit coupling on the phonon band structure of simple cubic Po is first shown to be the opposite of that on simple metals: as the initial Fermi surface (without SO) is strongly nested, the SO correction reduces the nesting and the resulting Kohn anomalies, and hardens most phonon frequencies. The inclusion of SO effects is essential to the correct representation of the phonons. Because of the delicate bonding characteristics of Po, whose lowtemperature simple cubic phase is stabilized by relativistic effects, increasing the temperature has the completely counterintuitive effect of stabilizing a less symmetric phase (rhombohedral $\beta$-Po). This transition originates in the competition of the $0 \mathrm{~K}$ total energy difference (including relativistic stabilizing effects), which favors the $\alpha$ phase, and slightly more favorable vibrational entropy (at higher temperatures) for the $\beta$ phase. A number of other elements are likely to have temperature behaviors similar to Po: either due to relativistic or pressure effects, complex bonding environments open the way for radically different symmetry breaking as a function of temperature, when entropic effects are of the same order of magnitude as the energy differences between bonding types.

The author wishes to thank M. Šob, N. Helbig, and O. Eriksson for useful discussions. This research has been supported by the Belgian FNRS, the EU FP6 project DNA Nanodevices (G.A. 029192), and the EU FP7 through the ETSF I3 e-I3 project (G. A. 211956). Computer time was provided by UCLouvain-CISM, CINES, and the Red Española de Supercomputacion.

*matthieu.jean.verstraete@gmail.com

[1] A. R. Oganov et al., Nature (London) 457, 863 (2009).

[2] T. Matsuoka and K. Shimizu, Nature (London) 458, 186 (2009).

[3] Y. Ma et al., Nature (London) 458, 182 (2009).

[4] W. L. Mao et al., Science 302, 425 (2003).

[5] E.Y. Tonkov, High Pressure Phase Transformations (Gordon and Breach, Philadelphia, 1992).

[6] W. H. Beamer and C. E. Maxwell, J. Chem. Phys. 17, 1293 (1949).

[7] M. I. McMahon and R. J. Nelmes, Chem. Soc. Rev. 35, 943 (2006).

[8] C. E. Maxwell, J. Chem. Phys. 17, 1288 (1949).

[9] D. Legut, M. Friák, and M. Šob, Phys. Rev. Lett. 99, 016402 (2007).

[10] B. I. Min et al., Phys. Rev. B 73, 132102 (2006).

[11] R.E. Kraig, D. Roundy, and M. L. Cohen, Solid State Commun. 129, 411 (2004).

[12] P. Karen et al., Phys. Today 61, No. 9, 10 (2008).

[13] R. M. Martin, Electronic Structure (Cambridge University Press, Cambridge, 2004).

[14] S. Baroni, S. de Gironcoli, A. Dal Corso, and P. Giannozzi, Rev. Mod. Phys. 73, 515 (2001).

[15] X. Gonze et al., Z. Kristallogr. 220, 558 (2005).

[16] W. Kohn and L. J. Sham, Phys. Rev. 140, A1133 (1965).

[17] C. Hartwigsen, S. Goedecker, and J. Hutter, Phys. Rev. B 58, 3641 (1998).

[18] A. Dal Corso, Phys. Rev. B 76, 054308 (2007).

[19] M. J. Verstraete et al., Phys. Rev. B 78, 045119 (2008).

[20] B. Grabowski, T. Hickel, and J. Neugebauer, Phys. Rev. B 76, 024309 (2007).

[21] W. Kohn, Phys. Rev. Lett. 2, 393 (1959).

[22] R. Brocklehurst, J. Goode, and L. Vassamillet, J. Chem. Phys. 27, 985 (1957).

[23] Lange's Handbook of Chemistry, edited by J. Dean (McGraw-Hill, New York, 1999), 15th ed.

[24] F. Murnaghan, Proc. Natl. Acad. Sci. U.S.A. 30, 244 (1944).

[25] P. Souvatzis, O. Eriksson, M. Katsnelson, and S. Rudin, Phys. Rev. Lett. 100, 095901 (2008).

[26] P. Zhang, Y. Xue, and P. Dev, Solid State Commun. 148, 151 (2008). 